\title{
A Better Approach for Solving a Fuzzy Multiobjective Programming Problem by Level Sets
}

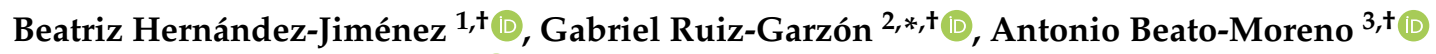 \\ and Rafaela Osuna-Gómez ${ }^{3,+}$ (D) \\ 1 Departamento de Economía, Métodos Cuantitativos e Historia Económica, Universidad Pablo de Olavide, \\ 41013 Sevilla, Spain; mbherjim@upo.es \\ 2 Instituto de Desarrollo Social y Sostenible (INDESS), Universidad de Cádiz, 11405 Jerez de la Frontera, Spain \\ 3 Departamento de Estadística e I.O., Universidad de Sevilla, 41004 Sevilla, Spain; beato@us.es (A.B.-M); \\ rafaela@us.es (R.O.-G.) \\ * Correspondence: gabriel.ruiz@uca.es \\ + These authors contributed equally to this work.
}

check for updates

Citation: Hernández-Jiménez, B.; Ruiz-Garzón, G.; Beato-Moreno, A.; Osuna-Gómez, R. A Better Approach for Solving a Fuzzy Multiobjective Programming Problem by Level Sets. Mathematics 2021, 9, 992. https:// doi.org/10.3390/math9090992

Academic Editor: Basil Papadopoulos

Received: 25 March 2021

Accepted: 25 April 2021

Published: 28 April 2021

Publisher's Note: MDPI stays neutral with regard to jurisdictional claims in published maps and institutional affiliations.

Copyright: (c) 2021 by the authors. Licensee MDPI, Basel, Switzerland. This article is an open access article distributed under the terms and conditions of the Creative Commons Attribution (CC BY) license (https:/ / creativecommons.org/licenses/by/ $4.0 /)$.
Abstract: In this paper, we deal with the resolution of a fuzzy multiobjective programming problem using the level sets optimization. We compare it to other optimization strategies studied until now and we propose an algorithm to identify possible Pareto efficient optimal solutions.

Keywords: multiple objective programming problems; fuzzy numbers; generalized Hukuhara differentiability

\section{Introduction}

The construction of scientific models to describe real situations represents a significant advance in the aid for decision-making, especially in complex problems. These models allow us to select the essential, discard the accessory, apply scientific knowledge, and, based on them, predict and compare the results of different strategies or decisions. They have proven especially useful when faced with the needs of optimal allocation of resources, evaluation of a system's performance, obtaining quantitative information for the improvement of processes or their limitations. The study of models where it is necessary to find and identify the best alternative among a collection of possibilities, without having to list and evaluate each explicitly and every one of them constitutes the Optimization Theory's objective. The situations that allow modeling, where the possible alternatives and the objectives can be expressed and quantified using mathematical functions, constitute a widely studied and applied line of knowledge, known as Mathematical Programming. In particular, Multiobjective Mathematical Programming is really useful in order to model real situations where more than one objective exists.

The growing use of optimization models to help decision-making, in fields as diverse and complex as Economics, Engineering, Social or Life Sciences, has created a demand for new tools that allow considering and solving more real situations, which, in general, do not verify the properties and hypotheses that for classical optimization are essential to ensure an effective search for solutions.

It is quite common to find a certain degree of incomplete, subjective, vague or inaccurate information in the situations to be modeled. The classic mathematical programming tools do not adequately reflect this lack of precision in the data that define both the objective functions and the constraints of the problem. When any of these types of inaccuracies appear in an optimization problem, it cannot be addressed with the classic mathematical optimization tools. Thus, the Fuzzy Set Theory was introduced to manage the imprecision or lack of accurate information that appears in many mathematical models or computations of some real-world phenomena. A significant and growing field is the Fuzzy Optimization that deals with the resolution of optimization problems with imprecise or vague data. 
Combining fuzzy and multiobjective optimization tools will help model real problems that cannot be modeled with classic optimization techniques. In the literature, since the first contribution in that sense (Zimmerman [1]), many works can be found where multiobjective optimization models are applied using fuzzy logic in general and fuzzy functions in particular to describe the real information [2-9].

The use of fuzzy logic in real life can be found in the digital display of some electrical appliances or in the brakes of a vehicle (Figures 1 and 2):

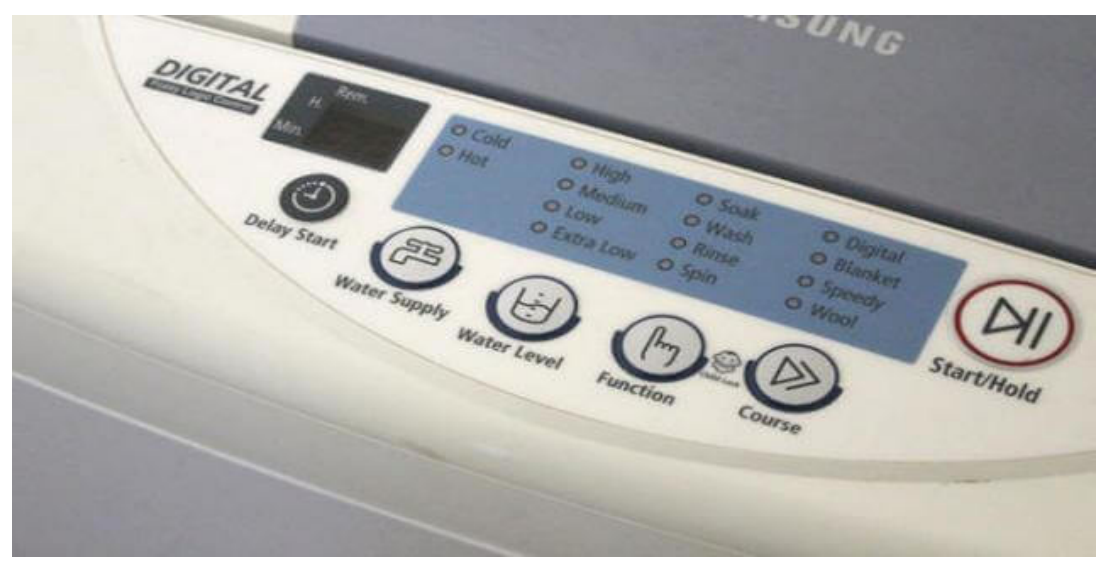

Figure 1. Digital display. Fuzzy logic control.

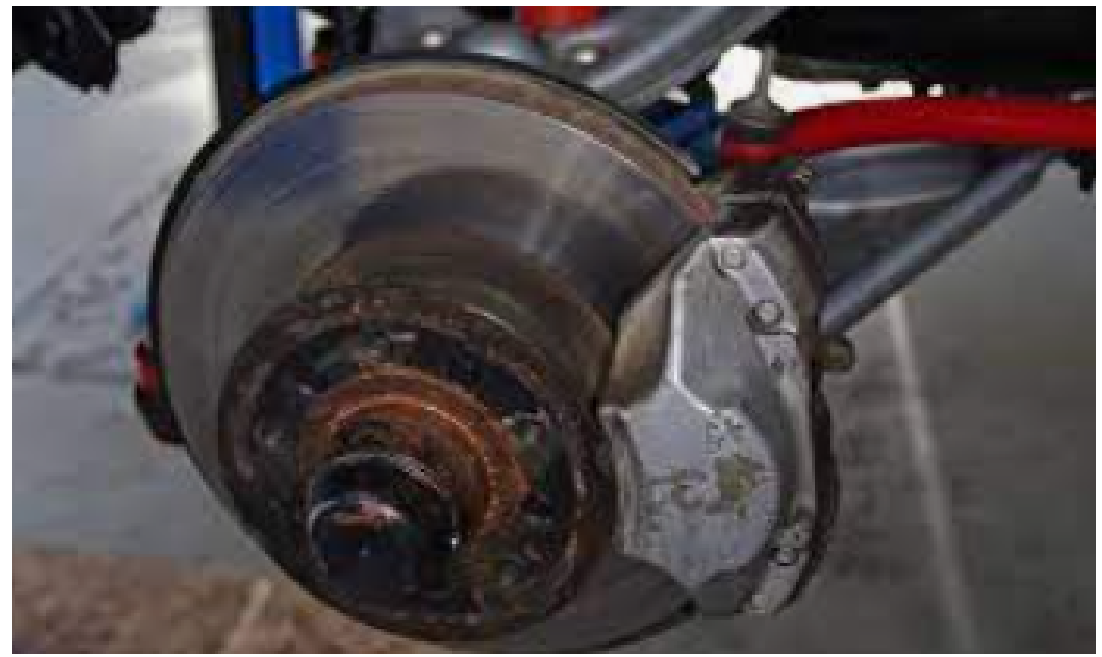

Figure 2. Fuzzy brake control system.

In $[10,11]$, the author uses the embedding theorem, i.e., the approximation of a fuzzy set by a crisp number (defuzzification techniques), to define the optimum concept and the scalarization in vector optimization to solve fuzzy multiobjective programming problems. However, when we use a defuzzification operator that replaces a fuzzy set by a single number, we generally lose too much important information.

In [12], an approximation of a fuzzy set by an interval is proposed. In this approach, a given fuzzy set is substituted by a crisp interval, which is-in some sense-close to the former one. A new interval approximation operator, the Nearest Interval Approximation Operator (NIA), is among interval approximation operators of a fuzzy number, the one that minimizes a certain measure of the distance to the fuzzy number. In [13], the authors explain how interesting it is to use the Nearest Interval Approximation Operator to solve a multiobjective programming problem with fuzzy objective functions, to reflect reality and have excellent computational behavior. They established a sufficient Karush-Kuhn-Tucker type of Pareto optimality conditions, using continuously gH-differentiable functions where 
the sum of the end-points functions is convex. This idea has been used in the literature in other works, for example, in [14] for solving fuzzy bilevel programming problems and in [15] for solving fully fuzzy multiobjective linear programming problems.

In [16], sufficient optimality conditions were obtained to solve a fuzzy multiobjective problem but not necessary conditions. It is well known that, to be sure you can find all the possible optimums for the optimization problem, the necessary optimality conditions can be developed since you cannot ensured it only with sufficient ones.

In this paper, we propose to use an optimality concept by levels, which means we will use the level sets that characterize the fuzzy set entirely to define the optimum [17]. We demonstrate that this concept is more general than the aforementioned. Undoubtedly, the optimum notion should be determined by the decider's interest, so, in some cases, the crisp optima or interval-optima can be considered more satisfactory than the ones showed here. However, by including the latter in the previous, the search mechanisms proposed here will be valid whatever the concept used.

Necessary conditions are introduced to identify the optimal candidates, using more general hypotheses than those existing in the literature. It is an advantage in the sense that they can be applied to a more significant number of problems. From a theoretical and practical point of view, the resolution of a differentiable optimization problem involves the application of necessary and sufficient optimality conditions, which characterize the optimality of the problems and serve as a basis for the design of efficient algorithms for the resolution of these problems. Recently, classical optimality conditions have been extended to different equilibrium, variational, or control problems in different spaces [18-22].

We obtain a multiobjective necessary optimality condition that, in the particular case, you have a single objective function that coincides with the one proposed by Osuna-Gomez et al. [23], and it has specially computational checking advantages. In addition, an algorithm to examine all possible candidates to be multiobjective optimal solutions under the only hypothesis in which the fuzzy functions are $g H$-differentiable functions is proposed.

This paper is organized as follows. In Section 2, we recall the order and arithmetic for using intervals and fuzzy numbers on $\alpha$-level sets. In Section 3, we focus on the multiobjective problem with fuzzy objective functions, defining the solution concept based on the $\alpha$-level sets, obtaining a Pareto-type solution concept and comparing it to the ones existing in the literature. In Section 4, the fundamental results on gH-differentiability of fuzzy functions are compiled and used to obtain optimality conditions. In Section 5, we prove our main results: a necessary optimality condition and an algorithm based on it. Finally, in Section 6, we present the Conclusions.

We can establish a sequencing of contents (Figure 3):

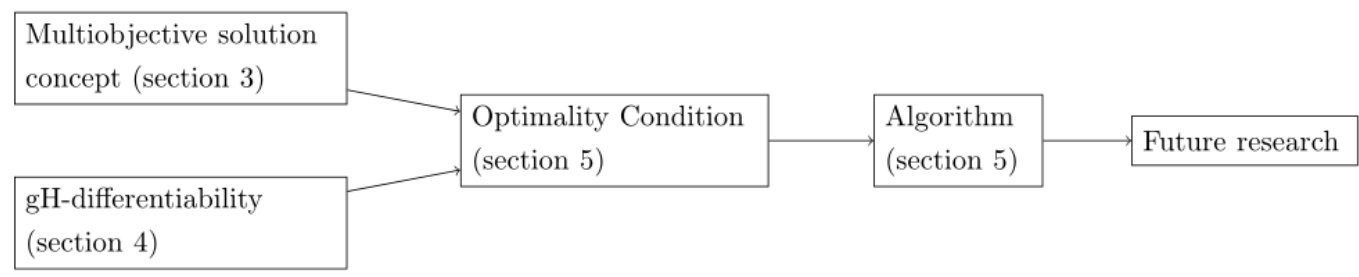

Figure 3. Sequencing of contents.

\section{Preliminaries}

We recall the arithmetic and order for intervals we are going to use.

We denote by $\mathcal{K}_{C}$ the family of all bounded closed intervals in $\mathbb{R}$, i.e.,

$\mathcal{K}_{C}=\{[\underline{a}, \bar{a}] / \underline{a}, \bar{a} \in \mathbb{R}$ and $\underline{a} \leq \bar{a}\}$.

For $A=[\underline{a}, \bar{a}], B=[\underline{b}, \bar{b}] \in \mathcal{K}_{C}$ and $v \in \mathbb{R}$, we consider the following operations:

$$
A+B=[\underline{a}, \bar{a}]+[\underline{b}, \bar{b}]=[\underline{a}+\underline{b}, \bar{a}+\bar{b}], \quad v A=v[\underline{a}, \bar{a}]= \begin{cases}{[v \underline{a}, v \bar{a}]} & \text { if } \quad v \geq 0 \\ {[v \bar{a}, v \underline{a}]} & \text { if } \quad v<0\end{cases}
$$




$$
A \ominus_{g H} B=C \Leftrightarrow\left\{\begin{array}{ll}
(a) & A=B+C, \\
(b) & B=A+(-1) C .
\end{array} \quad\right. \text { or }
$$

This difference (2), called generalized Hukuhara difference ( $g H$-difference for short), has many interesting properties compared to other definitions (Minskowki, Hukuhara differences) for example $A \ominus_{g H} A=\{0\}=[0,0]$. In addition, the $g H$-difference of two intervals $A=[\underline{a}, \bar{a}]$ and $B=[\underline{b}, \bar{b}]$ always exists, and it is equal to [24]

$$
A \ominus_{g H} B=[\min \{\underline{a}-\underline{b}, \bar{a}-\bar{b}\}, \max \{\underline{a}-\underline{b}, \bar{a}-\bar{b}\}] \text {. }
$$

Given two intervals, we define the distance between $A$ and $B$ by $H(A, B)=\max$ $\{|\underline{a}-\underline{b}|,|\bar{a}-\bar{b}|\}$. It is well-known that $\left(\mathcal{K}_{C}, H\right)$ is a complete metric space.

We consider the following order relation in $\mathcal{K}_{C}$.

Definition 1. Let $A=[\underline{a}, \bar{a}], B=[\underline{b}, \bar{b}] \in \mathcal{K}_{C}$. It is said that

- $\quad A \preceq B \Leftrightarrow \underline{a} \leq \underline{b}$ and $\bar{a} \leq \bar{b}$.

- $\quad A \preceq B \Leftrightarrow A \preceq B$ and $A \neq B$, i.e. $\underline{a} \leq \underline{b}$ and $\bar{a} \leq \bar{b}$, with a strict inequality.

- $\quad A \prec B \Leftrightarrow \underline{a}<\underline{\bar{b}}$ and $\bar{a}<\bar{b}$.

It is clear that $A \prec B \Rightarrow A \preceq B \Rightarrow A \preceq B$.

A fuzzy set on $\mathbb{R}^{n}$ is a mapping $\tilde{u}: \overline{\mathbb{R}}^{n} \rightarrow[0,1]$. For each fuzzy set $\tilde{u}$, we denote its $\alpha$-level set as $[\tilde{u}]^{\alpha}=\left\{x \in \mathbb{R}^{n} \mid \tilde{u}(x) \geq \alpha\right\}$ for any $\alpha \in(0,1]$. The support of $\tilde{u}$ is denoted by $\operatorname{supp}(\tilde{u})$ where $\operatorname{supp}(\tilde{u})=\left\{x \in \mathbb{R}^{n} \mid \tilde{u}(x)>0\right\}$. The closure of $\operatorname{supp}(\tilde{u})$ is defined by the 0-level of $u$, i.e., $[\tilde{u}]^{0}=\operatorname{cl}(\operatorname{supp}(\tilde{u}))$, where $\operatorname{cl}(M)$ means the closure of the subset $M \subset \mathbb{R}^{n}$. The core of $\tilde{u}, \operatorname{core}(\tilde{u})$, is defined by $\operatorname{core}(\tilde{u})=\left\{x \in \mathbb{R}^{n} \mid \tilde{u}(x)=1\right\}$.

Definition 2. A fuzzy set on $\mathbb{R}, \tilde{u}: \mathbb{R} \rightarrow[0,1]$ is said to be a fuzzy interval or fuzzy number if the following properties are satisfied:

1. $\tilde{u}$ is normal, i.e., there exists $x_{0} \in \mathbb{R}$ such that $\tilde{u}\left(x_{0}\right)=1$;

2. $\tilde{u}$ is an upper semi-continuous function;

3. $\tilde{u}(\lambda x+(1-\lambda) y) \geq \min \{\tilde{u}(x), \tilde{u}(y)\}, x, y \in \mathbb{R}, \lambda \in[0,1]$;

4. $[\tilde{u}]^{0}$ is compact.

Let $\mathcal{F}_{C}$ denote the family of all fuzzy intervals on $\mathbb{R}$. Thus, for any $\tilde{u} \in \mathcal{F}_{C}$, we have that $[\tilde{u}]^{\alpha} \in \mathcal{K}_{C}$ for all $\alpha \in[0,1]$, and we denote its $\alpha$-levels by $[\tilde{u}]^{\alpha}=\left[\underline{u}_{\alpha}, \bar{u}_{\alpha}\right]$, for all $\alpha \in[0,1]$. A fuzzy interval is completely determined by $[\tilde{u}]^{\alpha}=\left[\underline{u}_{\alpha}, \bar{u}_{\alpha}\right]$ satisfying certain conditions, [17].

Triangular fuzzy numbers are a special type of fuzzy numbers which are well determined by three real numbers $a \leq b \leq c$. We write $\tilde{b}=(a, b, c)$ to denote the triangular fuzzy number $\tilde{b}$ with core or 1-level given by the singleton $\{b\}$ and whose $\alpha$-levels sets are

$$
[\tilde{b}]^{\alpha}=[a+(b-a) \alpha, c-(c-b) \alpha],
$$

for all $\alpha \in[0,1]$. A particular case of triangular fuzzy number (or fuzzy number) are the real numbers $a \in \mathbb{R}$ with a membership function given by $\chi_{\{a\}}$, where $\chi_{A}$ denotes the characteristic function of the set $A$. In addition, considering the characteristic function, we can see that any interval $A=[\underline{a}, \bar{a}]$ is a fuzzy interval, i.e., $\chi_{A}$ is a fuzzy interval (fuzzy number) such that $\left[\chi_{A}\right]^{\alpha}=A$, for all $\alpha \in[0,1]$.

For fuzzy numbers, $\tilde{u}, \tilde{v} \in \mathcal{F}_{C}$, represented by $\left[\underline{u}_{\alpha}, \bar{u}_{\alpha}\right]$ and $\left[\underline{v}_{\alpha}, \bar{v}_{\alpha}\right]$ respectively, and, for any real number $\lambda$, we define the addition $\tilde{u}+\tilde{v}$ and scalar multiplication $\lambda \tilde{u}$ as follows:

$$
(\tilde{u}+\tilde{v})(x)=\sup _{y+z=x} \min \{\tilde{u}(y), \tilde{v}(z)\}, \quad(\lambda \tilde{u})(x)= \begin{cases}\tilde{u}\left(\frac{x}{\lambda}\right), & \text { if } \lambda \neq 0 \\ 0, & \text { if } \lambda=0\end{cases}
$$


It is well known that, for every $\alpha \in[0,1]$,

$$
\begin{gathered}
{[\tilde{u}+\tilde{v}]^{\alpha}=\left[(\underline{u+v})_{\alpha},(\overline{u+v})_{\alpha}\right]=\left[\underline{u}_{\alpha}+\underline{v}_{\alpha}, \bar{u}_{\alpha}+\bar{v}_{\alpha}\right],} \\
{[\lambda \tilde{u}]^{\alpha}=\left[(\underline{\lambda u})_{\alpha},(\overline{\lambda u})_{\alpha}\right]=\lambda[\tilde{u}]^{\alpha}=\lambda\left[\underline{u}_{\alpha}, \bar{u}_{\alpha}\right]=\left[\min \left\{\lambda \underline{u}_{\alpha}, \lambda \bar{u}_{\alpha}\right\}, \max \left\{\lambda \underline{u}_{\alpha}, \lambda \bar{u}_{\alpha}\right\}\right] .}
\end{gathered}
$$

Definition 3 ([25]). Given two fuzzy intervals $\tilde{u}$ and $\tilde{v}$, the generalized Hukuhara difference ( $g$ H-difference for short) is the fuzzy interval $\tilde{w}$, if it exists, such that

$$
\tilde{u} \ominus_{g H} \tilde{v}=\tilde{w} \Leftrightarrow \begin{cases} & \text { (i) } \tilde{u}=\tilde{v}+\tilde{w}, \\ \text { or } & \text { (ii) } \tilde{v}=\tilde{u}+(-1) \tilde{w} .\end{cases}
$$

It is easy to show that $(i)$ and $(i i)$ are both valid if and only if $w$ is a crisp number. Note that the case (i) coincides with Hukuhara difference (see [26]), and so the $g H$-difference concept is more general than the $H$-difference one.

If $\tilde{u} \ominus_{g H} \tilde{v}$ exists, then, in terms of $\alpha$-level sets, we have that

$$
\left[\tilde{u} \ominus_{g H} \tilde{v}\right]^{\alpha}=[\tilde{u}]^{\alpha} \ominus_{g H}[\tilde{v}]^{\alpha}=\left[\min \left\{\underline{u}_{\alpha}-\underline{v}_{\alpha}\right\}, \max \left\{\bar{u}_{\alpha}-\bar{v}_{\alpha}\right\}\right]
$$

for all $\alpha \in[0,1]$, where $[u]^{\alpha} \ominus_{g H}[v]^{\alpha}$ denotes the gH-difference between two intervals (see [25]).

Given $\tilde{u}, \tilde{v} \in \mathcal{F}_{C}$, the distance between $\tilde{u}$ and $\tilde{v}$ is defined by

$$
D(\tilde{u}, \tilde{v})=\sup _{\alpha \in[0,1]} H\left([u]^{\alpha},[v]^{\alpha}\right)=\sup _{\alpha \in[0,1]} \max \left\{\left|\underline{u}_{\alpha}-\underline{v}_{\alpha}\right|,\left|\bar{u}_{\alpha}-\bar{v}_{\alpha}\right|\right\} .
$$

Thus, $\left(\mathcal{F}_{C}, D\right)$ is a metric space.

Let us now consider the set of all vector fuzzy intervals, $\mathcal{F}_{C}^{m}$, i.e., $\tilde{u} \in \mathcal{F}_{C}^{m}$ if $\tilde{u}=$ $\left(\tilde{u}_{1}, \ldots, \tilde{u}_{m}\right)$, where each $\tilde{u}_{i} \in \mathcal{F}_{C}$. For any $\tilde{u} \in \mathcal{F}_{C}^{m}$, we have that

$$
[\tilde{u}]^{\alpha}=\left\{x=\left(x_{1}, \ldots, x_{m}\right) \in \mathbb{R}^{m} \mid \tilde{u}_{i}\left(x_{i}\right) \geq \alpha, \quad i=1, \ldots, m\right\} .
$$

Geometrically, it would be a Cartesian product of closed intervals in $\mathbb{R}^{m}$

$$
[\tilde{u}]^{\alpha}=\prod_{i=1}^{m}\left[\tilde{u}_{i}\right]^{\alpha} \text {. }
$$

Lemma 1. It is verified that $[\tilde{u}]^{\alpha} \subseteq[u]^{0}$, for all $\alpha \in[0,1], \forall \tilde{u} \in \mathcal{F}_{C}^{m}$.

Proof. If $x \in[\tilde{u}]^{\alpha}$, then $\tilde{u}_{i}\left(x_{i}\right) \geq \alpha \geq 0$, for all $i=1, \ldots m$ and then $x \in[\tilde{u}]^{0}$.

We recall the usual order for fuzzy intervals based on $\alpha$-level sets:

Definition 4. For $\tilde{u}, \tilde{v} \in \mathcal{F}_{C}$, it is said that:

- $\quad \tilde{u} \supseteqq \tilde{v}$, if for every $\alpha \in[0,1],[\tilde{u}]^{\alpha} \supseteqq[\tilde{v}]^{\alpha}$.

If $\tilde{u} \preceq \tilde{v}, \tilde{v} \supseteqq \tilde{u}$, then $\tilde{u}=\tilde{v}$.

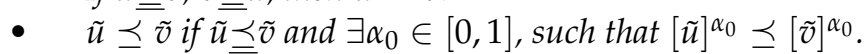

- $\quad \tilde{u} \prec \tilde{v}$ if $\left[\overline{\bar{u}]^{\alpha}} \prec[\tilde{v}]^{\alpha}, \forall \alpha \in[0,1]\right.$.

For $\tilde{u}, \tilde{v} \in \mathcal{F}_{C}$ if either $\tilde{u} \preceq \tilde{v}$ or $\tilde{v} \leqq \tilde{u}$, then it is said that $\tilde{u}$ and $\tilde{v}$ are comparable; otherwise, they are incomparable.

Note that $\preceq$ is a partial order relation on $\mathcal{F}_{C}$. Thus, $\tilde{v} \succeq \tilde{u}$ instead of $\tilde{u} \preceq \tilde{v}$ can be written. We observe that, if $\tilde{u} \prec \tilde{v}$, then $\tilde{u} \preceq \tilde{v}$ and then $\tilde{u} \preceq \tilde{v}$. 
Henceforth, $S$ denotes an open subset of $\mathbb{R}$. Let us consider $\tilde{f}: S \rightarrow \mathcal{F}_{C}$ a fuzzy function or fuzzy mapping. For each $\alpha \in[0,1]$, we associate with $\tilde{f}$ the interval-valued functions family $\tilde{f}_{\alpha}: S \rightarrow \mathcal{K}_{C}$ given by $\tilde{f}_{\alpha}(t)=[\tilde{f}(t)]^{\alpha}$. For any $\alpha \in[0,1]$, we denote

$$
\tilde{f}_{\alpha}(t)=\left[\underline{f}_{\alpha}(t), \bar{f}_{\alpha}(t)\right]=[\underline{f}(\alpha, t), \bar{f}(\alpha, t)] .
$$

Here, for each $\alpha \in[0,1]$, the real-valued endpoint functions $\underline{-}_{\alpha^{\prime}} \bar{f}_{\alpha}: S \rightarrow \mathbb{R}$ are called lower and upper functions of $\tilde{f}$, respectively.

\section{Multiobjective Problem with Fuzzy Objective Functions}

In this paper, we consider the following multiobjective fuzzy mathematical programming problem:

$$
\text { (P) } \quad \operatorname{Min}\left(\tilde{f}_{1}(x), \ldots, \tilde{f}_{p}(x)\right)
$$

where $\tilde{f}_{j}: S \rightarrow \mathcal{F}_{C}, j=1, \ldots, p$ are fuzzy functions defined on $S$, an open non-empty subset in $\mathbb{R}$.

Interpreting the meaning of "minimize a vector fuzzy function," we give a similar solution concept to the non-dominated solution than the one introduced by Pareto, and usually considered in real-valued multiobjective optimization.

Definition 5. Let $\tilde{f}: S \rightarrow \mathcal{F}_{C}^{p}$ be a vector fuzzy function defined on $S$. It is said that $x^{*} \in S$ is an efficient solution or Pareto solution if there exists no $x \in S$ such that $\tilde{f}_{j}(x) \leqq \tilde{f}_{j}\left(x^{*}\right), \forall j=1, \ldots, p$ and $\exists k$ such that $\tilde{f}_{k}(x) \prec \tilde{f}_{k}\left(x^{*}\right)$.

Notice that the aforementioned definition coincides with the classic one when the functions are real-valued.

In [10], a defuzzification function, $\eta: \mathcal{F}_{C} \rightarrow \mathbb{R}$ and two order relations are defined

Definition 6. Let $\tilde{u}$ and $\tilde{v}$ be fuzzy numbers. We write

- $\quad \tilde{u} \preceq^{1} \tilde{v}$ if the Hukuhara difference $\tilde{v} \ominus_{H} \tilde{u}$ exists and $\eta\left(\tilde{v} \ominus_{H} \tilde{u}\right) \geq 0$.

- $\quad \tilde{u} \preceq^{2} \tilde{v}$ if the Hukuhara difference $\tilde{v} \ominus_{H} \tilde{u}$ exists and $\tilde{v} \ominus_{H} \tilde{u}$ is nonnegative, where a fuzzy number $\tilde{a}$ is said to be nonnegative if $\underline{a}_{\alpha} \geq 0$ for all $\alpha \in[0,1]$.

Notice that these definitions can only be applied to fuzzy numbers such that their Hukuhara difference exists, i.e., $\mu\left([\tilde{u}]^{\alpha}\right) \leq \mu\left([\tilde{v}]^{\alpha}\right), \forall \alpha \in[0,1]$, where $\mu$ is the interval range.

Now, we are going to relate those order relations on $\mathcal{F}_{C}$ with the one we propose to use "ฏ":

Proposition 1. $\preceq^{2}$ and $\preceq$ coincide when Hukuhara difference does exist.

Proof. If $A, B \in \mathcal{K}_{C}, A \preceq B$ if and only if $A \ominus_{g H} B \preceq[0,0]$ because

$$
A \ominus_{g H} B=[\min \{\underline{a}-\underline{b}, \bar{a}-\bar{b}\}, \max \{\underline{a}-\underline{b}, \bar{a}-\bar{b}\}] .
$$

If $A \preceq B$, then $\underline{a}-\underline{b} \leq 0$ and $\bar{a}-\bar{b} \leq 0$. Thus, $A \ominus_{g H} B \preceq[0,0]$ and reciprocally.

In particular, if the Hukuhara difference does exist, then $\tilde{v} \ominus_{H} \tilde{u}=\tilde{v} \ominus_{g H} \tilde{u}$. If $\tilde{v} \ominus_{g H} \tilde{u}$ is nonnegative $[0,0] \preceq\left[\tilde{v} \ominus_{g H} \tilde{u}\right]^{\alpha}$ for all $\alpha \in[0,1]$. From (5), $[0,0] \preceq[\tilde{v}]^{\alpha} \ominus_{g H}[\tilde{u}]^{\alpha}$, therefore $[\tilde{u}]^{\alpha} \leqq[\tilde{v}]^{\alpha}$ and $\tilde{u} \preceq \tilde{v}$ from Definition 4 .

In [23], it is shown that a minimum based on average index ordering relation is a minimum for $\alpha$-level sets ordering relation when $\eta(\tilde{u})=\int_{Y} \lambda_{\underline{u}_{\alpha}}+(1-\lambda) \bar{u}_{\alpha} d P(\alpha)$, where $Y$ is a subset of the unit interval and $P$ a probability distribution function on $Y$. In general:

Proposition 2. If $\eta(\tilde{a}) \geq 0$ when $\tilde{a}$ is a nonnegative fuzzy number, then $\varliminf^{1}$ is equivalent to 
Proof. Let us suppose that $\tilde{v} \ominus_{H} \tilde{u}$ exists, then $\tilde{u} \preceq^{1} \tilde{v} \Leftrightarrow \eta\left(\tilde{v} \ominus_{H} \tilde{u}\right) \geq 0 \Leftrightarrow \eta\left(\tilde{v} \ominus_{g H} \tilde{u}\right) \geq$ $0 \Leftrightarrow \tilde{v} \ominus_{g H} \tilde{u}$ is nonnegative $\Leftrightarrow[0,0] \preceq\left[\tilde{v} \ominus_{g H} \tilde{u}\right]^{\alpha}=[\tilde{v}]^{\alpha} \ominus_{g H}[\tilde{u}]^{\alpha}$ forall $\alpha \in[0,1] \Leftrightarrow \tilde{u} \preceq \tilde{v}$.

Definition 7. An interval approximation of a fuzzy number is an operator $I: \mathcal{F}_{C} \rightarrow \mathcal{K}_{C}$ such that, for $\tilde{u}, \tilde{v} \in \mathcal{F}_{C}$,

(i) $I(\tilde{u}) \subset \operatorname{supp}(\tilde{u})$,

(ii) $\operatorname{core}(\tilde{u}) \subset I(\tilde{u})$,

(iii) $\forall \epsilon>0, \exists \delta>0 / D(\tilde{u}, \tilde{v})<\delta \Rightarrow H(I(\tilde{u}), I(\tilde{v}))<\epsilon$.

Proposition 3 ([12]). The interval

$$
N(\tilde{u})=\left[\int_{0}^{1} \underline{u}(x, \alpha) d \alpha, \int_{0}^{1} \bar{u}(x, \alpha) d \alpha\right]
$$

is an Interval Approximation of fuzzy number $\tilde{u}$ such that $N(\tilde{u})$ minimizes $D(\tilde{u}, I(\tilde{u}))$ for all I belonging to the space of interval approximation operators of fuzzy numbers. $N(\cdot)$ is called Nearest Interval Approximation (NIA).

Notice that $D(\tilde{u}, I(\tilde{u}))$ is the distance between fuzzy numbers, defined in $\mathcal{F}_{C}$, and it can be considered here since each interval is also a fuzzy number with constant $\alpha$-level sets for all $\alpha \in[0,1]$.

In [13] to find a solution of $(P)$, the authors have approximated it by the following interval multiobjective program:

$$
\text { (P1) } \quad \operatorname{Min}\left(F_{1}(x), \ldots, F_{p}(x)\right)
$$

where $F_{j}(x)$ stands for the nearest interval approximation (NIA) of $\tilde{f}_{j}(x)$, i.e., $F_{j}(x)=$ $\left[\int_{0}^{1} f_{j}(x, \alpha) d \alpha, \int_{0}^{1} \bar{f}_{j}(x, \alpha) d \alpha\right], x \in S$ that it is among the interval approximation operators of a fuzzy number, the one that minimizes the distance to the fuzzy number.

Definition 8 ([13]). $x^{*} \in S$ is called a satisficing solution of $(P)$ if it is a Pareto optimal solution of $(P 1)$, i.e., if there is no $x \in S$ such that $F_{j}(x) \preceq F_{j}\left(x^{*}\right)$ for all $j=1, \ldots, p$ and $F(x) \neq F\left(x^{*}\right)$.

Proposition 4. If $x^{*}$ is a satisficing solution of $(P)$, then $x^{*}$ is a Pareto solution for $(P)$.

Proof. Let us suppose that there exists $x \in S$ such that $\tilde{f}_{j}(x) \preceq \tilde{f}_{j}\left(x^{*}\right)$. Then, it follows that $\underline{f}_{j}(x, \alpha) \leq \underline{f}_{j}\left(x^{*}, \alpha\right)$ and $\bar{f}_{j}(x, \alpha) \leq \bar{f}_{j}\left(x^{*}, \alpha\right), \forall \alpha$ and thus $F_{j}(x) \leq F_{j}\left(x^{*}\right)$ and besides there exists $k$ such that $f_{k}(x, \alpha)<f_{k}\left(x^{*}, \alpha\right)$ and $\bar{f}_{k}(x, \alpha)<\bar{f}_{k}\left(x^{*}, \alpha\right), \forall \alpha$ and thus $F_{k}(x)<F_{k}\left(x^{*}\right)$. It stands in contradiction to the hypothesis.

In this way, it is demonstrated that the Pareto solution concept used in this work includes as particular cases those existing in the literature.

\section{4. gH-Differentiable Fuzzy Functions}

In Optimization Theory, when the functions are differentiable, their gradients allow us to find analytical optimality conditions. Different fuzzy differentiability concepts have been used in fuzzy optimization (H-differentiability, G-differentiability, level-wise differentiability), but they are more restrictive than the $g H$-differentiability [27-29].

Next, we present the $g H$-differentiable fuzzy function concept based on the $g H$ difference of fuzzy numbers.

Definition 9 ([30]). The $g H$-derivative of a fuzzy function $\tilde{f}: S \rightarrow \mathcal{F}_{C}$ at $t_{0} \in S$ is defined as

$$
\tilde{f}^{\prime}\left(t_{0}\right)=\lim _{h \rightarrow 0} \frac{1}{h}\left[\tilde{f}\left(t_{0}+h\right) \ominus_{g H} \tilde{f}\left(t_{0}\right)\right]
$$


If $\tilde{f}^{\prime}\left(t_{0}\right) \in \mathcal{F}_{C}$ satisfying (6) exists, we say that $\tilde{f}$ is generalized Hukuhara differentiable ( $g$ H-differentiable, for short) at $t_{0}$.

The following results establish relationships between gH-differentiability of $\tilde{f}$ and the gH-differentiability of the interval-valued functions family $\tilde{f}_{\alpha}$, and the differentiability of its endpoint functions $\underline{f}_{\alpha}$ and $\bar{f}_{\alpha}$.

Notation 1. For simplicity, we use the following notation for an interval. If $\alpha, \beta$ are the end-points of the interval $A \in \mathcal{K}_{C}$ (but it is not necessarily $\alpha \leq \beta$ ), we write $A=[\alpha \bigvee \beta]$.

Theorem 1 ([31]). If $\tilde{f}: S \rightarrow \mathcal{F}_{C}$ is $g H$-differentiable at $t_{0} \in S$, then $\tilde{f}_{\alpha}$ is $g H$-differentiable at $t_{0}$ uniformly in $\alpha \in[0,1]$ and

$$
\tilde{f}_{\alpha}^{\prime}\left(t_{0}\right)=\left[\tilde{f}^{\prime}\left(t_{0}\right)\right]^{\alpha},
$$

for all $\alpha \in[0,1]$.

From Theorem 1 and using Theorem 8 given by Qiu [32], it is verified that

Theorem 2. Let $\tilde{f}: S \rightarrow \mathcal{F}_{C}$ be a fuzzy function. If $\tilde{f}$ is $g H$-differentiable at $t_{0} \in S$, then the lateral derivatives of real-valued endpoints functions $\left(\underline{f}_{\alpha}\right)_{-}^{\prime}\left(t_{0}\right),\left(\underline{f}_{\alpha}\right)_{+}^{\prime}\left(t_{0}\right),\left(\bar{f}_{\alpha}\right)_{-}^{\prime}\left(t_{0}\right)$ and $\left(\bar{f}_{\alpha}\right)_{+}^{\prime}\left(t_{0}\right)$ exist, uniformly in $\alpha \in[0,1]$, and satisfy

$$
\left[\tilde{f}^{\prime}\left(t_{0}\right)\right]^{\alpha}=\tilde{f}_{\alpha}^{\prime}\left(t_{0}\right)=\left[\left(\underline{f}_{\alpha}\right)_{+}^{\prime}\left(t_{0}\right) \bigvee\left(\bar{f}_{\alpha}\right)_{+}^{\prime}\left(t_{0}\right)\right] .
$$

Remark 1. As a consequence of the aforementioned results, we use the following notation:

$$
\left[\tilde{f}^{\prime}\left(t_{0}\right)\right]^{\alpha}=\tilde{f}_{\alpha}^{\prime}\left(t_{0}\right)=\left[\left(\tilde{f}_{\alpha}^{\prime}\left(t_{0}\right)\right)^{L},\left(\tilde{f}_{\alpha}^{\prime}\left(t_{0}\right)\right)^{U}\right]
$$

where

$$
\begin{aligned}
& \left(\tilde{f}_{\alpha}^{\prime}\left(t_{0}\right)\right)^{L}=\min \left\{\left(\underline{f}_{\alpha}\right)_{+}^{\prime}\left(t_{0}\right),\left(\bar{f}_{\alpha}\right)_{+}^{\prime}\left(t_{0}\right)\right\}=\min \left\{\left(\underline{f}_{\alpha}\right)_{-}^{\prime}\left(t_{0}\right),\left(\bar{f}_{\alpha}\right)_{-}^{\prime}\left(t_{0}\right)\right\}, \\
& \left(\tilde{f}_{\alpha}^{\prime}\left(t_{0}\right)\right)^{U}=\max \left\{\left(\underline{f}_{\alpha}\right)_{+}^{\prime}\left(t_{0}\right),\left(\bar{f}_{\alpha}\right)_{+}^{\prime}\left(t_{0}\right)\right\}=\max \left\{\left(\underline{f}_{\alpha}\right)_{-}^{\prime}\left(t_{0}\right),\left(\bar{f}_{\alpha}\right)_{-}^{\prime}\left(t_{0}\right)\right\} .
\end{aligned}
$$

Definition 10. Given a fuzzy vector function $\tilde{f}=\left(\tilde{f}_{1}, \ldots, \tilde{f}_{p}\right) \in \mathcal{F}_{C}^{p}$, we say that $\tilde{f}$ is a vector $g H$-differentiable fuzzy function at $t_{0} \in S$ if and only if $\tilde{f}_{j}$ is $g H$-differentiable at $t_{0}$, for all $j=1, \ldots, p$.

\section{Necessary Conditions for Pareto Solutions}

In this section, we prove a necessary optimality condition for Pareto solutions and give an algorithm based on it, in order to find all the potential candidates to be optimum for our problem. With the necessary optimality conditions, we can exclude feasible solutions that are not optimums. In Section 3, we have proved that optimum concepts used until now in the different strategies to solve a fuzzy multiobjective programming problem are more restrictive than the Pareto solution concept based on the $\alpha$-level sets. Thus, the algorithm we develop here remains valid for the other optimum notions.

In [16], the same Pareto efficiency notion we propose in this paper is used, and a sufficient optimality conditions for it is proved, but using more restrictive hypotheses on the functions (level-wise differentiable functions) than the ones we suppose here. Thus, the necessary optimality condition presented here and the algorithm developed remain valid in the context of that paper [16]. 
Notation 2. For convenience, we introduce the following notations. Let $A=\left(A_{1}, \ldots, A_{p}\right)$ be with $A_{j} \in \mathcal{K}_{C}$. Letting $\Lambda \in \mathcal{M}^{p \times 2}$, we denote by $\Lambda \times A$ the lineal combination

$$
\Lambda \times A=\sum_{j=1}^{p} \lambda_{j 1} \underline{a}_{j}+\lambda_{j 2} \bar{a}_{j}
$$

If $0 \in A_{j}$ for some $j=1, \ldots, p$, then there exists $\Lambda \in \mathcal{M}^{p \times 2}, \lambda_{j i} \geq 0$ and not all zero, such that: $\Lambda \times A=0$.

If $\Lambda \times A=0$ with $\lambda_{j i} \geq 0$, but not all zero, there exist $\underline{\lambda}, \bar{\lambda},(\underline{\lambda}, \bar{\lambda}) \geq 0$ such that

$$
0 \in\left[\underline{\lambda}^{T} \underline{a}, \bar{\lambda}^{T} \bar{a}\right]
$$

with $\underline{a}=\left(\underline{a}_{1}, \ldots, \underline{a}_{p}\right)$ and $\bar{a}=\left(\bar{a}_{1}, \ldots, \bar{a}_{p}\right)$.

Proposition 5. Let $\tilde{f}$ be a vector $g H$-differentiable fuzzy function on $S$. If $x^{*} \in S$ is an efficient or Pareto solution for $(P)$, then the following system does not have a solution at $y \in \mathbb{R}$ :

$$
y\left(\tilde{f}_{j}^{\prime}\left(x^{*}\right)\right) \prec \tilde{0}^{p} \quad j=1, \ldots, p .
$$

Proof. Arguing by contradiction, let us suppose that $\exists y \in \mathbb{R}$ such that

$$
y \tilde{f}_{j}^{\prime}\left(x^{*}\right) \prec \tilde{0} \quad \forall j=1, \ldots p .
$$

Then,

$$
y\left[\tilde{f}_{j}^{\prime}\left(x^{*}\right)\right]^{\alpha} \prec[0,0] \quad \forall \alpha \in[0,1] \quad \forall j=1, \ldots, p .
$$

From Theorem 2, for each $j$, there exist $\left(f_{j}\left(\alpha,\left(x^{*}\right)\right)_{+}^{\prime}\right.$ and $\left(\overline{f_{j}}\left(\alpha, x^{*}\right)\right)_{+}^{\prime}$, uniformly in $\alpha \in[0,1]$, and they satisfy

$$
\left[\tilde{f}_{j}^{\prime}\left(x^{*}\right)\right]^{\alpha}=\left[\min \left\{\left(\underline{f_{j}}\left(\alpha, x^{*}\right)\right)_{+}^{\prime},\left(\overline{f_{j}}\left(\alpha, x^{*}\right)\right)_{+}^{\prime}\right\}, \max \left\{\left(\underline{f_{j}}\left(\alpha, x^{*}\right)\right)_{+,}^{\prime}\left(\overline{f_{j}}\left(\alpha, x^{*}\right)\right)_{+}^{\prime}\right\}\right]
$$

Since

$$
y\left[f_{j}^{\prime}\left(x^{*}\right)\right]^{\alpha} \prec[0,0] \Leftrightarrow\left\{\begin{array}{l}
y\left(\overline{f_{j}}\left(\alpha, x^{*}\right)\right)_{+}^{\prime}<0 \\
y\left(\underline{f_{j}}\left(\alpha, x^{*}\right)\right)_{+}^{\prime}<0
\end{array}, \quad \forall \alpha \in[0,1] .\right.
$$

From

$$
y\left(\underline{f}_{j}\left(\alpha, x^{*}\right)\right)_{+}^{\prime}=\lim _{t \rightarrow 0^{+}} \frac{1}{t}\left(\underline{f_{j}}\left(\alpha, x^{*}+y t\right)-\underline{f_{j}}\left(\alpha, x^{*}\right)\right)<0
$$

Thus, it follows that there exist $\underline{\epsilon_{j}^{+}}>0$, such that, for all $t$, with $0<t<\underline{\epsilon_{j}^{+}}$

$$
\begin{gathered}
\underline{f_{j}}\left(\alpha, x^{*}+y t\right)-\underline{f_{j}}\left(\alpha, x^{*}\right)<0 \quad \forall \alpha \in[0,1], \\
\underline{f_{j}}\left(\alpha, x^{*}\right)-\underline{f_{j}}\left(\alpha, x^{*}\right)<0, \quad \forall \alpha \in[0,1] .
\end{gathered}
$$

In addition, analogously there exists $\overline{\epsilon_{j}^{+}}>0$ such that, for all $t$, with $0<t<\overline{\epsilon_{j}^{+}}$

$$
\begin{gathered}
\overline{f_{j}}\left(\alpha, x^{*}+y t\right)-\overline{f_{j}}\left(\alpha, x^{*}\right)<0 \quad \forall \alpha \in[0,1], \\
\overline{f_{j}}\left(\alpha, x^{*}\right)-\overline{f_{j}}\left(\alpha, x^{*}\right)<0, \quad \forall \alpha \in[0,1] .
\end{gathered}
$$

Taking $\epsilon=\min \left\{\epsilon_{j}^{+}, \overline{\epsilon_{j}^{+}}: \quad j=1, \ldots, p\right\}, t \in(0, \epsilon)$ and from (8) and (9):

$$
\underline{f}(\alpha, x)-\underline{f}\left(\alpha, x^{*}\right)<0 \quad \text { and } \quad \bar{f}(\alpha, x)-\bar{f}\left(\alpha, x^{*}\right)<0, \quad \forall \alpha \in[0,1],
$$


where we suppose that $x \in S$. Hence, $\exists x \in S$ and $\tilde{f}(x) \prec \tilde{f}\left(x^{*}\right)$, and this is a contradiction to $x^{*}$ is an efficient solution for $\tilde{f}$.

Now, we prove the main result of this section.

Theorem 3. Let $\tilde{f}: S \rightarrow \mathcal{F}_{C}^{p}$ be a vector $g H$-differentiable fuzzy function at $x^{*} \in S$. If $x^{*}$ is an efficient or Pareto solution for $(P)$, then there exists a non-negative matrix $\Lambda \in \mathcal{M}^{p \times 2}$ such that

$$
\Lambda \times\left[\tilde{f}^{\prime}\left(x^{*}\right)\right]^{0}=0 .
$$

Proof. If $x^{*}$ is a Pareto solution for $\tilde{f}$, then (7) does not have a solution. From Lemma 1,

$$
\begin{gathered}
y\left[\tilde{f}^{\prime}\left(x^{*}\right)\right]^{\alpha} \prec[0,0], \quad \forall \alpha \in[0,1] \quad \forall j=1, \ldots, p \Leftrightarrow \\
y\left[\tilde{f}_{j}^{\prime}\left(x^{*}\right)\right]^{0} \prec[0,0] \quad \forall j=1, \ldots, p .
\end{gathered}
$$

Now, let us consider the following lineal system and let us prove it has no solution:

$$
\left\{\begin{array}{l}
y A<0 \\
y B<0
\end{array}\right.
$$

where $A, B \in \mathcal{M}^{p \times 1}$ and

$$
A=\left(\begin{array}{c}
\left(\left(\tilde{f}_{1}^{\prime}\right)_{0}\left(x^{*}\right)\right)^{L} \\
\vdots \\
\left(\left(\tilde{f}_{p}^{\prime}\right)_{0}\left(x^{*}\right)\right)^{L}
\end{array}\right), \quad B=\left(\begin{array}{c}
\left(\left(\tilde{f}_{1}^{\prime}\right)_{0}\left(x^{*}\right)\right)^{U} \\
\vdots \\
\left(\left(\tilde{f}_{p}^{\prime}\right)_{0}\left(x^{*}\right)\right)^{U}
\end{array}\right) .
$$

If (11) has a solution, then the system (7) also would have a solution, and this is impossible by Proposition 5.

Since (11) is a system of linear inequalities and it does not have solution, from Gordan's alternative theorem, there exist $\alpha, \beta \in \mathbb{R}^{p}$ with $\alpha \geqq 0, \beta \geqq 0$ but not all zero, such that

$$
A^{T} \alpha+B^{T} \beta=0 \Leftrightarrow \sum_{j=1}^{p} \alpha_{j}\left(\left(\tilde{f}_{j}^{\prime}\right)_{0}\left(x^{*}\right)\right)^{L}+\beta_{j}\left(\left(\tilde{f}_{j}^{\prime}\right)_{0}\left(x^{*}\right)\right)^{U}=0 .
$$

Redefining $\Lambda=\left(\alpha_{j}, \beta_{j}\right)$, we obtain that there exists $\Lambda \in \mathcal{M}^{p \times 2}$ such that

$$
\Lambda \times\left[\tilde{f}^{\prime}\left(x^{*}\right)\right]^{0}=0 .
$$

In addition, the proof is completed.

Remark 2. If there exists $j=1, \ldots, p$ such that $0 \in\left[\tilde{f}_{j}^{\prime}\left(x^{*}\right)\right]^{0}$, (12) is verified, and then, to identify possible candidates for Pareto solutions, is reduced to identify those feasible solutions whose 0-level set of the derivative contains the zero element. This coincides with the result for a unique objective function given by Osuna-Gómez et al. [23].

Remark 3. Expression (12) is equivalent to the existence of a positive linear combination of

$$
\left\{\left(\underline{f}_{j}^{\prime}\right)_{+}\left(x^{*}, 0\right),\left(\overline{f^{\prime}}\right)_{+}\left(x^{*}, 0\right), j=1, \ldots, p\right\}
$$

equal to zero. This means that (12) is equivalent to the existence of $\underline{\lambda}, \bar{\lambda} \in \mathbb{R}^{p}, \underline{\lambda}, \bar{\lambda} \geqq 0$ not both zero such that

$$
\underline{\lambda}^{T}\left(\tilde{f}^{\prime}\right)_{0}^{L}\left(x^{*}\right)+\bar{\lambda}^{T}\left(\tilde{f}^{\prime}\right)_{0}^{U}\left(x^{*}\right)=0 .
$$


From Theorems 2, 3 and Remark 3, an algorithm to identify the Pareto solutions candidates can be designed, in the same form as in classical Mathematical Programming. We solve the equations where the gradients are equal to zero in order to identify the possible optima.

The corresponding flow chart is (Figure 4):

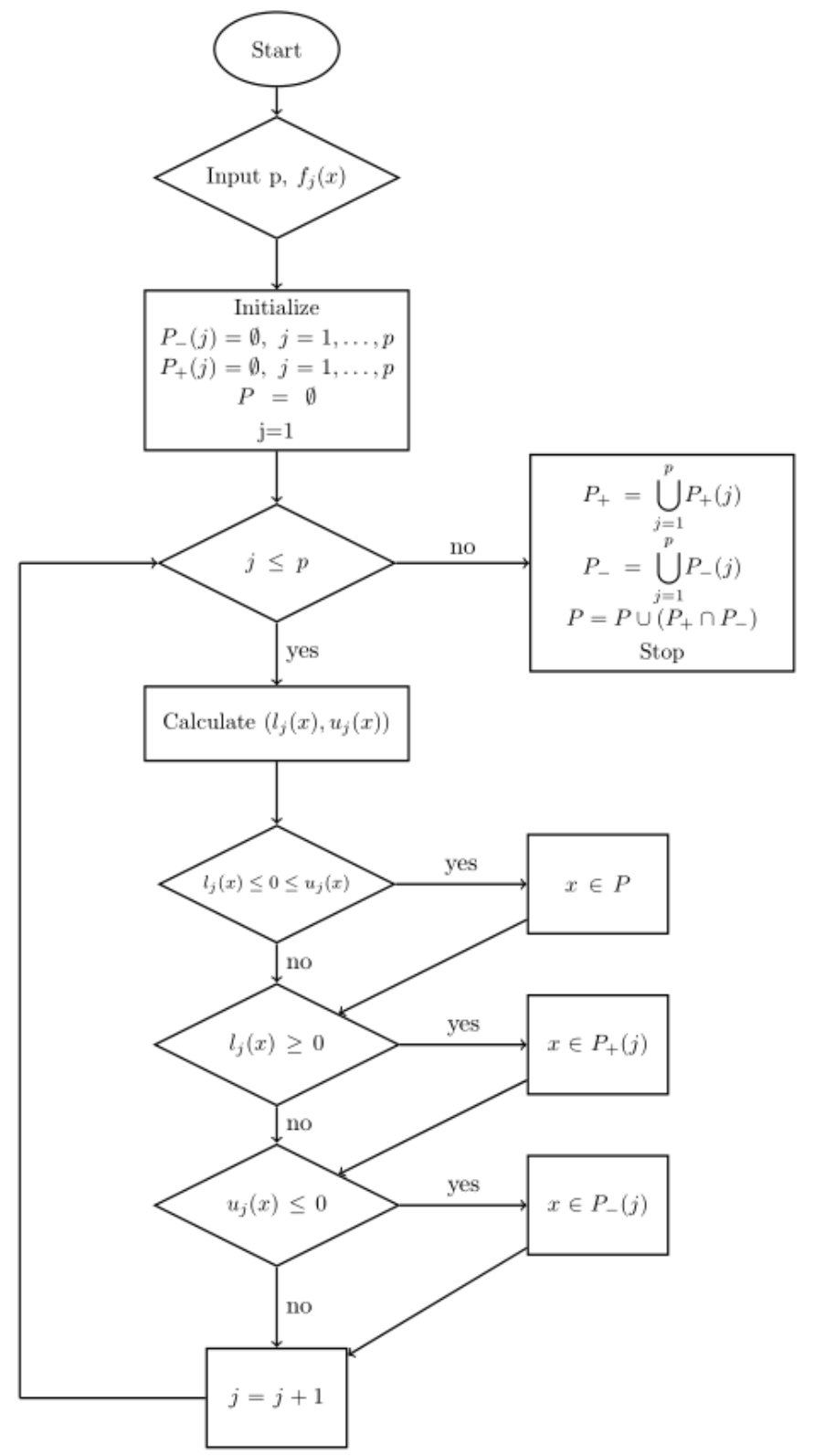

Figure 4. Flow chart.

The algorithm can be described as:

Step 1. Start and input $p$ (Objective function $\left(\tilde{f}_{1}(x), \ldots, \tilde{f}_{p}(x)\right)$ )

Step 2. Put $j=1, P=\varnothing, P_{+}=\varnothing, P_{-}=\varnothing$

Step 3. While $j \leq p$

Step $3.1 P_{+}(j)=P_{-}(j)=\varnothing$.

Step 3.2 Calculate $\underline{f}_{j}^{\prime}(x, 0)_{+}, \bar{f}_{j}^{\prime}(x, 0)_{+}$

Step 3.3 Define and find $l_{j}(x)=\min \left\{\underline{f}_{j}^{\prime}(x, 0)_{+}, \bar{f}_{j}^{\prime}(x, 0)_{+}\right\}$,

$u_{j}(x)=\max \left\{\underline{f}_{j}^{\prime}(x, 0)_{+}, \bar{f}_{j}^{\prime}(x, 0)_{+}\right\}$ 
Step 3.4 Put $\left[l_{j}(x), u_{j}(x)\right]$

Step 3.5 For $x$ such that $l_{j}(x) \leq 0 \leq u_{j}(x)$, then $x \in P$.

Step 3.6 For $x$ such that $l_{j}(x) \geq 0$, then $x \in P_{+}(j)$

Step 3.7 For $x$ such that $u_{j}(x) \leq 0$, then $x \in P_{-}(j)$

Step $3.8 j=j+1$

Step 4. Set $P_{+}=\bigcup_{j=1}^{p} P_{+}(j)$ and $P_{-}=\bigcup_{j=1}^{p} P_{-}(j)$. If $x \in P_{+} \cap P_{-}$then $x \in P$

Step 5. Print $P=$ possible Pareto solutions set and stop.

Example 1. We look for the Pareto optimal solutions for

$$
\text { (P) } \operatorname{Min}\left(\tilde{f}_{1}(x), \tilde{f}_{2}(x)\right), x \in \mathbb{R}
$$

- In step 1 , let us consider two fuzzy functions $\tilde{f}_{1}, \tilde{f}_{2}: \mathbb{R} \rightarrow \mathcal{F}_{C}$, whose $\alpha$-level sets are given by

$$
\left[\tilde{f}_{1}(x)\right]^{\alpha}=\left[\left(x-2+\alpha,(x-\alpha)^{2}\right],\left[\tilde{f}_{2}(x)\right]^{\alpha}=[1+\alpha, 2(3-\alpha)] x, \alpha \in[0,1]\right.
$$

, respectively, because $\tilde{f}_{2}(x)=C x$, where $C$ is a fuzzy interval.

- In step 2 , for $j=1$, we begin with $P=\varnothing, P_{+}=\varnothing$ and $P_{-}=\varnothing$.

- In step 3, for $j=1,\left[\tilde{f}_{1}^{\prime}(x)\right]^{0}=[\min \{2 x-4,2 x\}, \max \{2 x-4,2 x\}]$.

Then, $l_{1}(x)=2 x-4 \leq 0 \leq u_{1}(x)=2 x$, so we get that $P=[0,2]$ is a possible Pareto efficient solution for $(P), P_{+}(1)=[2,+\infty)$ and $P_{-}(1)=(-\infty, 0]$

For $j=2,\left[\tilde{f}_{2}^{\prime}(x)\right]^{0}=[1,6]$ and so $P=\varnothing, P_{+}(2)=\mathbb{R}$ and $P_{-}(2)=\varnothing$

- $\quad$ In Step $4, P_{+}=P_{+}(1) \cup P_{+}(2)=\mathbb{R}$ and $P_{-}=P_{-}(1) \cup P_{-}(2)=(-\infty, 0]$

- In step 5 , in summary, we get that $x \in(-\infty, 2]$ is a possible Pareto efficient solution for $(P)$. It is represented in the following Figure 5:

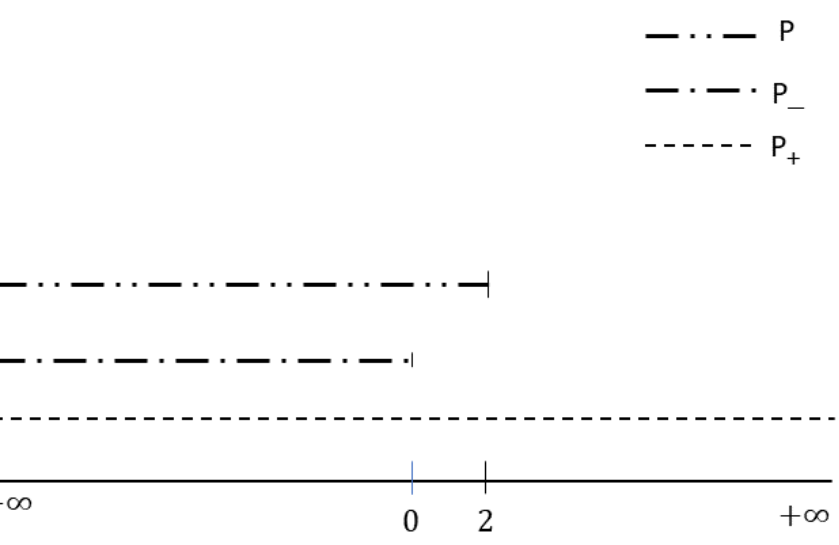

Figure 5. Pareto solutions set.

\section{Conclusions}

In this paper, we have addressed the resolution of a fuzzy multiobjective programming problem using the $\alpha$-level sets optimization. We have obtained a necessary condition for Pareto-type solutions from the $\alpha$-level sets and have provided an algorithm and an example based on it.

The advantages of our method are focused on: the solution concept used, based on the $\alpha$-level sets, encompassing those previously presented in the literature; the algorithm proposed, that locates the optimal candidates easily and comfortably; the generalization to multiobjective fuzzy case of results obtained for fuzzy scalar problems and real-valued multiobjective problems. 
The disadvantages or limitations are the necessity of gH-differentiability for the involved functions, and the proposed algorithm does not allow for identifying the points that are not solutions.

In our opinion, future work should be based, on the one hand, on obtaining sufficient conditions that can identify optimal solutions to multiobjective problems, not only candidates for optimal solutions and that are generalizable to control and variational problems. On the other hand, future work should focus on exploring more complete algorithms (such as the bisection search method, Newton's method, the cyclic coordinate method, Rosenbrock's method, and others) based on the obtained optimality conditions that allow for the effective and practical search of the solutions.

Author Contributions: Writing-review and editing, B.H.-J., G.R.-G., A.B.-M. and R.O.-G. All authors have read and agreed to the published version of the manuscript.

Funding: This research received no external funding.

Acknowledgments: The authors would like to thank the referees for their suggestions that have improved the initial manuscript and the Institute for Social and Sustainable Development (INDESS) for the facilities provided for the preparation of this article.

Conflicts of Interest: The authors declare no conflict of interest.

\section{References}

1. Zimmerman, H.J. Fuzzy programming and linear programming with several objective functions. Fuzzy Sets Syst. 1978, 1, 45-55. [CrossRef]

2. Hu, Y.; Zhang, Y.; Gong, D.W. Multiobjective Particle Swarm Optimization for Feature Selection With Fuzzy Cost. IEEE Trans. Cybern. 2021, 51, 874-888. [CrossRef]

3. Jamwal, P.K.; Hussain, S. A fuzzy based multiobjective optimization of multi echelon supply chain network. J. Intell. Fuzzy Syst. 2020, 39, 3057-3066. [CrossRef]

4. Milenkovic, M.; Bojović, N. Fuzzy multiobjective rail freight car fleet composition. In Optimization Models for Rail Car Fleet Management; Elsevier, 2020. Available online: https:/ /www.sciencedirect.com/book/9780128151549/optimization-models-forrail-car-fleet-management (accessed on 25 March 2021).

5. Goli, A.; Zare, H.K.; Tavakkoli-Moghaddam, R.; Sadegheih, A. Multiobjective fuzzy mathematical model for a financially constrained closed-loop supply chain with labor employment. Comput. Intell. 2020, 36, 4-34. [CrossRef]

6. Sun, Y. A Fuzzy Multi-Objective Routing Model for Managing Hazardous Materials Door-to-Door Transportation in the Road-Rail Multimodal Network With Uncertain Demand and Improved Service Level. IEEE Access 2020, 8, 172808-172828. [CrossRef]

7. Birjandi, M.R.S.; Shahraki, F.; Razzaghi, K. Hydrogen and $\mathrm{CO}_{2}$ Management in the Refinery with Fuzzy Multiobjective Nonlinear Programming. Chem. Eng. Technol. 2019, 42, 1941-1951. [CrossRef]

8. Bigdeli, H.; Hassanpour, H.; Tayyebi, J. Multiobjective security game with fuzzy payoffs. Iran. J. Fuzzy Syst. 2019, 16, 89-101.

9. Upmanyu, M.; Saxena, R.R. On solving multi objective Set Covering Problem with imprecise linear fractional objectives. RAIROOper. Res. 2015, 49, 495-510. [CrossRef]

10. Wu, H.C. Solutions of fuzzy multiobjective programming problems based on the concept of scalarization. J. Optim. Theory Appl. 2008, 139, 361-378. [CrossRef]

11. Wu, H.C. Using the technique of scalarization to solve the multiobjective programming problems with fuzzy coefficients. Math. Comput. Model. 2008, 48, 232-248. [CrossRef]

12. Grzegorzewski, P. Nearest interval approximation of a fuzzy number. Fuzzy Sets Syst. 2002, 130, 321-330. [CrossRef]

13. Luhandjula, M.K.; Rangoaga, M.J. An approach for solving a fuzzy multiobjetive programming problem. Eur. J. Oper. Res. 2014, 232, 249-255. [CrossRef]

14. Ren, A.; Wang, Y. An approach for solving a fuzzy bilevel programming problem through nearest interval approximation approach and KKT optimality conditions. Soft Comput. 2017, 21, 5515-5526. [CrossRef]

15. Sharma, U.; Aggarwal, S. Solving fully fuzzy multi-objective linear programming problem using nearest interval approximation of fuzzy number and interval programming. Int. J. Fuzzy Syst. 2018, 20, 488-499. [CrossRef]

16. Wu, H.C. The optimality conditions for optimization problems with convex constraints and multiple fuzzy-valued objective functions. Fuzzy Optim. Decis. Mak. 2009, 9, 295-321. [CrossRef]

17. Goestschel, R.; Voxman, W. Elementary fuzzy calculus. Fuzzy Sets Syst. 1986, 18, 31-43. [CrossRef]

18. Ruiz-Garzón, G.; Osuna-Gómez, R.; Rufián-Lizana, A.; Hernández-Jiménez, B. Optimality and duality on Riemannian manifolds. Taiwan J. Math. 2018, 22, 1245-1259. [CrossRef]

19. Ruiz-Garzón, G.; Osuna-Gómez, R.; Ruiz-Zapatero, J. Necessary and sufficient optimality conditions for vector equilibrium problems on Hadamard manifolds. Symmetry 2019, 11, 1037. [CrossRef] 
20. Ruiz-Garzón, G.; Ruiz-Zapatero, J.; Osuna-Gómez, R.; Rufián-Lizana, A. Neccesary and sufficient second-order optimality conditions on Hadamard manifolds. Mathematics 2020, 8, 1152. [CrossRef]

21. Ruiz-Garzón, G.; Osuna-Gómez, R.; Rufián-Lizana, A.; Hernández-Jiménez, B. Approximate efficient solutions of the vector optimization problem on Hadamard manifolds via vector variational inequalities. Mathematics 2020, 8, 2196. [CrossRef]

22. Treanta, S.; Arana-Jiménez, M.; Antczak, T. A necessary and sufficient condition on the equivalence between local and global optimal solutions in variational control problems. Nonlinear Anal. 2020, 191, 111640. [CrossRef]

23. Osuna-Gómez, R.; Hernández-Jiménez, B.; Chalco-Cano, Y.; Ruiz-Garzón, G. Different optimum notions for fuzzy functions and optimality conditions associated. Fuzzy Optim. Decis. Mak. 2018, 17, 177-193. [CrossRef]

24. Stefanini, L.; Bede, B. Generalized Hukuhara differentiability of interval-valued functions and interval differential equations. Nonlinear Anal. 2009, 71, 1311-1328. [CrossRef]

25. Stefanini, L. A generalization of Hukuhara difference and division for interval and fuzzy arithmetic. Fuzzy Sets Syst. 2010, 161, 1564-1584. [CrossRef]

26. Hukuhara, M. Integration des applications mesurables dont la valeur est un compact convexe. Funkc. Ekvacioj 1967, 10, 205-223.

27. Stefanini, L.; Arana-Jiménez, M. On Fréchet and Gateaux derivatives for interval and fuzzy-valued functions in the setting of gH-differenciability. In Proceedings of the 2020 IEEE International Conference on Fuzzy Systems (FUZZ-IEEE), Glasgow, UK, 19-24 July 2020; pp. 1-6.

28. Puri, M.L.; Ralescu, D.A. Differentials for fuzzy functions. J. Math. Anal. Appl. 1983, 91, 552-558. [CrossRef]

29. Bede, B.; Gal, S.G. Generalizations of the differentiability of fuzzy number valued functions with applications to fuzzy differential equation. Fuzzy Sets Syst. 2005, 151, 581-599. [CrossRef]

30. Bede, B.; Stefanini, L. Generalized differentiability of fuzzy-valued functions. Fuzzy Sets Syst. 2013, 230, 119-141. [CrossRef]

31. Chalco-Cano, Y.; Rodríguez-López, R.; Jiménez-Gamero, M.D. Characterizations of generalized differentiable fuzzy functions. Fuzzy Sets Syst. 2016, 295, 37-56. [CrossRef]

32. Qiu, D. The generalized Hukuhara differentiability of interval-valued function is not fully equivalent to the one-sided differentiability of its endpoint functions. Fuzzy Sets Syst. 2020. [CrossRef] 